10

\title{
Differential Nonlinear Absorption of an Elliptically Polarized Femtosecond Vortex Beam in Tellurite Glass*
}

\author{
(C) A.M. Alshehria ${ }^{1}$, El Sayed Yousefa ${ }^{1}$, A.A. Alshahrania ${ }^{1}$, Akram Ibrahima ${ }^{1}$, Nafis Ahmada ${ }^{1}$, and V.R. Bhardwajb ${ }^{2}$ \\ ${ }^{1}$ Department of Physics, King Khalid University, \\ P.O. Box 9004, Abha, Saudi Arabia \\ ${ }^{2}$ Department of Physics, University of Ottawa, Advanced Research Complex, \\ Ottawa, Canada \\ e-mail:
}

Received October 20, 2019

Revised January 25, 2020

Accepted March 15, 2020

We present differential nonlinear absorption of an elliptically polarized femtosecond laser vortex beam carrying an angular momentum of $l=-1$ in tellurite glass. Transmission measurements were utilized to measure the nonlinear absorption of the glass on a shot-by-shot basis, and to investigate the modification threshold for the beam. Additionally, the effect of pulse energy on the polarization ellipse was investigated. The results revealed a reduction in the ellipticity as the incident pulse energy was increased. Non-rotation of the ellipsoid indicated that there is no change in the third order nonlinear susceptibility $\left(\chi^{(3)}\right)$ of the telluride glass upon irradiation by the femtosecond beam. The transmission of multiple pulses through the same spot revealed that although an effect was evident for the first ten pulses, the subsequent pulses had no effect. The transmission of a complete cycle of increasing and decreasing pulse energies revealed no hysteresis of the femtosecond laser vortex beam in tellurite glass in contrast to that reported for Gaussian beams in silica glass.

Keywords: vortex beam, tellurite glass, nonlinear absorption.

* Полный текст статьи опубликован в „Optics and Spectroscopy“ 2020 V. 128. N 8. 